\title{
BI-RADS 3-5 microcalcifications can preoperatively predict breast cancer HER2 and Luminal a molecular subtype
}

\author{
DongZhi Cen ${ }^{1, *}$, Li Xu ${ }^{2, *}$, Ningna $\mathrm{Li}^{2, *}$, Zhiguang Chen ${ }^{2, *}$, Lu Wang ${ }^{2, *}$, Shuqin Zhou ${ }^{2, *}$, Biao \\ $\mathbf{X u}^{2, *}$, Chun ling Liư ${ }^{3}$, Zaiyi Liu ${ }^{3}$, Tingting Luo ${ }^{4}$ \\ ${ }^{1}$ Department of Radiation Oncology and Department of Nuclear Medicine, The Third Affiliated Hospital of Guangzhou Medical \\ University, Guangzhou, 510150, Guangdong Province, People's Republic of China \\ ${ }^{2}$ Guangdong Provincial Traditional Chinese Medicine Hospital, Guangzhou, Guangdong Province 510120, P.R. China \\ ${ }^{3}$ Department of Radiology, Guangdong General Hospital, Guangdong Academy of Medical Sciences, Guangzhou, Guangdong \\ Province 510080, People's Republic of China \\ ${ }^{4}$ Department of Ultrasound, The Third People's Hospital of Shenzhen, Guangdong Shenzhen 518112, China \\ *These authors contributed equally to this work
}

Correspondence to: Zaiyi Liu, email: zyliu@163.com

Keywords: calcification, infiltrating ductal carcinoma, mammography, logistic regression, breast cancer molecular subtype Received: September 20, 2016

Accepted: January 07, 2017

Published: January 14, 2017

\section{ABSTRACT}

Purpose: To investigate associations between breast cancer molecular subtype and the patterns of mammographically detected calcifications.

Results: Identified were $93(19.1 \%)$ Luminal A, 242 (49.9\%) Luminal B, $108(22.2 \%)$ HER2 and $42(8.7 \%)$ basal subtypes. In univariate analysis, the clinicopathological parameters and BI-RADS 3-5 microcalcifications, which consisted 9 selected features was significantly associated with breast cancer molecular subtype (all $P<0.05$ ). Among subtypes, multivariate analysis showed that calcification $>2 \mathrm{~cm}$ in range (OR: $1.878,95 \% \mathrm{CI}: 1.150$ to 3.067 ) and calcification $>0.5 \mathrm{~mm}$ in diameter (OR:2.206, 95\% CI: 1.235 to 3.323 ) was independently predictive of HER2 subtype. The model showed good discrimination for predicting HER2 subtype, with a C-index of 0.704. In addition, multivariate analysis showed that calcification morphology (amorphour or coarse heterogenous calcifications OR: 2.847, 95\% CI: 1.526 to 5.312) was independently predictive of Luminal A subtype. The model showed good discrimination for predicting Luminal A subtype, with a C-index of 0.74 . And we demonstrated that amorphour or coarse heterogenous calcifications were associated with a higher incidence of Luminal A subtype than pleomorphic or fine linear or branching calcifications. There was no significant difference between breast cancer subtypes (Luminal B vs. other; Basal vs. other) and the patterns of mammographically detected calcifications.

Materials and Methods: Mammographic images of 485 female patients were included. The correlation between mammographic imaging features and breast cancer subtype was analyzed using Chi-square test, univariate and binary logistic regression analysis.

Conclusions: This study shows that BI-RADS 3-5 microcalcifications can be conveniently used to facilitate the preoperative prediction of HER2 and Luminal A molecular subtype in patients with infiltrating ductal carcinoma.

\section{INTRODUCTION}

Worldwide, the most common invasive female cancer observed is breast cancer [1]. Molecular subtyping of breast cancer tissue samples has become a common practice for individualized disease management, elucidation of disease prognosis, and avoidance of overtreatment [2]. Breast cancer molecular subtypes based on immunohistochemical (IHC) markers include Luminal A/B, HER2, and basal-like [3]. Clinical differences 
have been recognized among these genetically distinct tumor. [3, 4] For example, relative to Luminal A-positive samples, HER-2-positive neoplasms were found to have nearly 2.0 times the likelihood of having four or more metastatic lymph nodes and 1.6 times the likelihood of having multifocal disease [3]. The characteristic features of Luminal A tumors include stage-1 disease, lymph node negativity, and well-differentiated cells, whereas those of Luminal B tumors include HER-2-positive nonluminal cancers with a high tumor grade, lymph-nodal metastases, robust proliferation, and advanced-stage disease [5].

Characteristic imaging phenotypes have been associated with the aforementioned three breast cancer molecular subtypes. The basal-like subtype has been associated with non-calcified, circumscribed masses with posterior acoustic enhancement. Luminal A/B subtype tumors are often spiculated masses with a poorly circumscribed margin. And HER2-enriched tumors have been observed to have pleomorphic calcifications [6]. Reported studies have used various factors to predict breast cancer molecular subtype such as genes associated with histopathologic features [7]; the MHC class II (MHC II) antigen presentation pathway [8]; features extracted from magnetic resonance images $[9,10,11]$ and ultrasound features [2].

With the spread of screening mammography, microcalcifications have become a commonly observed positive sign of possible cancer [12]. The ability to predict molecular subtype reliably based on mammography findings would be helpful for treatment planning [13]. To the best of our knowledge, there is no literature that has determined whether a calcification features would enable superior prediction of breast cancer molecular subtype in invasive ductal carcinoma of breast. Therefore, the aim of this study was to investigate associations between breast cancer tumor molecular subtype and the patterns of mammographically detected calcifications.

\section{RESULTS}

Hierarchical clustering yielded distinct groups of gene expression trends and patterns of mammographically detected calcifications (Figure 1). Breast cancers in the 485 patients were classified into molecular subtypes as follows: 93 (19.2\%) Luminal A; 242 (49.9\%) Luminal B; 108 (22.3\%) HER2; and 42 (8.7\%) Basal. In 485 patients, the average age was $51.7 \pm 11.0$ years (standard deviation). The average tumor size (from the pathology reports) was $2.1 \mathrm{~cm} \pm 1.2$. We graded the tumors as follows: $6.2 \%$ (30/485), low grade; $59.9 \%$ (264/441), intermediate grade; and 43.3\% (191/441), high grade (Table 1).

In univariate analysis, the clinicopathological parameters and BI-RADS 3-5 microcalcification categories, which consisted 9 selected features (grade: $\chi^{2}=98.527$; tumor size: $\chi^{2}=23.063$; lymph node metastasis: $\chi^{2}=8.852$; lymphovascular invasion: $\chi^{2}=13.177$; Feature A: $\chi^{2}=23.531$; Feature B: $\chi^{2}=15.618$; Feature C: $\chi^{2}=20.149$; Feature D: $\chi^{2}=23.094$; Feature $\mathrm{E}: \chi^{2}=11.858$, Table 2 ), were associated with particular cancer molecular subtypes (all $P<0.05$ ).

Multivariate analysis showed that calcification with a range $>2 \mathrm{~cm}$ (OR: $1.87895 \%$ CI: $1.150-3.067$ ) or calcification with a diameter $>0.5 \mathrm{~mm}$ (OR: $2.20695 \%$ CI: 1.235-3.323) were predictive of the HER2 subtype (Figure 2). The model showed good discrimination for prediction of the HER2 subtype (C-index: 0.704). In addition, multivariate analysis showed that calcification morphology (amorphour or coarse heterogenous calcifications OR: 2.847 95\% CI: 1.526-5.312; Figure 3) was independently predictive of Luminal A subtype (C-index: 0.74). And we demonstrated that amorphour or coarse heterogenous calcifications were associated with a higher incidence of Luminal A subtype than pleomorphic or fine linear or branching calcifications. We did not detect significant differences in imaged calcification patterns among the breast cancer subtypes (Luminal B vs.

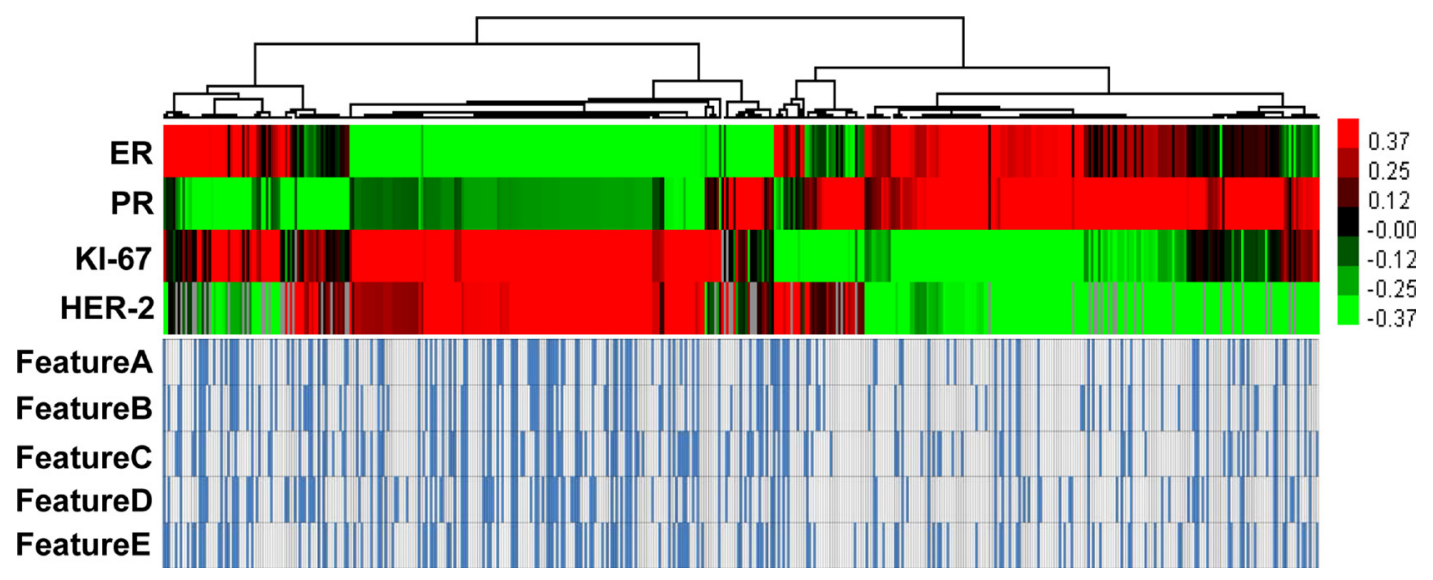

Figure 1: Hierarchical clustering yielded distinct groups of gene expression trends and patterns of mammographically detected calcifications. 


\begin{tabular}{|c|c|c|c|c|c|c|}
\hline & \multicolumn{4}{|c|}{ Molecular subtype } & \multicolumn{2}{|c|}{ Pearson $\chi^{2}$ test } \\
\hline & Luminal A & Luminal B & $\begin{array}{c}\text { HER-2 } \\
\text { enriched }\end{array}$ & Basal & $\chi^{2}$ & $P$ value \\
\hline Age (years) & & & & & 6.903 & $0.328 \dagger$ \\
\hline$<35$ & $7(7.5)$ & $17(7.0)$ & $3(2.8)$ & $3(7.1)$ & & \\
\hline $35-69$ & $77(82.8)$ & $214(88.4)$ & $99(91.7)$ & $35(83.3)$ & & \\
\hline$\geq 70$ & $9(9.7)$ & $11(4.5)$ & $6(5.6)$ & $4(9.5)$ & & \\
\hline Grade & & & & & 98.527 & $<0.001 \dagger$ \\
\hline grade 1 & $20(21.5)$ & $9(3.7)$ & $1(0.9)$ & $0(0)$ & & \\
\hline grade 2 & $65(69.9)$ & $142(58.7)$ & $42(38.9)$ & $15(25.7)$ & & \\
\hline grade 3 & $8(8.6)$ & $91(37.6)$ & $65(60.2)$ & $27(64.3)$ & & \\
\hline Tumor Size & & & & & 23.063 & $0.002 \dagger$ \\
\hline $\mathrm{T} 1$ & $59(69.4)$ & $112(50.9)$ & $36(37.5)$ & $17(53.1)$ & & \\
\hline $\mathrm{T} 2$ & $26(30.6)$ & $104(47.3)$ & $55(57.3)$ & $15(46.9)$ & & \\
\hline $\mathrm{T} 3$ & $0(0)$ & $4(1.8)$ & $5(5.2)$ & 0() & & \\
\hline Lymph node metastasis & & & & & 8.852 & 0.031 \\
\hline Negative & $45(67.2)$ & $98(48.8)$ & $52(55.9)$ & $20(66.7)$ & & \\
\hline Positive & $22(32.8)$ & $103(51.2)$ & $41(44.1)$ & $10(33.3)$ & & \\
\hline Lymphovascular invasion & & & & & 13.177 & 0.004 \\
\hline Negative & $78(86.7)$ & $161(69.4)$ & $74(71.8)$ & $35(85.4)$ & & \\
\hline Positive & $12(13.3)$ & $71(30.6)$ & $29(28,2)$ & $6(14.6)$ & & \\
\hline Feature A (Morphology) & & & & & 23.531 & $<0.001$ \\
\hline Amorphour, Coarse heterogenous & $80(86.0)$ & $173(71.5)$ & $61(56.5)$ & $34(81.0)$ & & \\
\hline Pleomorphic, Fine linear or branching, Combined & $13(14.0)$ & $69(28.5)$ & $47(43.5)$ & $8(19.0)$ & & \\
\hline Feature B (Distribution) & & & & & 15.618 & 0.001 \\
\hline Grouped or Clustered & 79 (84.9) & $170(70.2)$ & $65(60.2)$ & $32(76.2)$ & & \\
\hline Linear, Segmental & $14(15.1)$ & $72(29.8)$ & $43(39.8)$ & $10(23.8)$ & & \\
\hline Feature C (Range) & & & & & 20.149 & $<0.001$ \\
\hline$<2 \mathrm{~cm}$ & $75(80.6)$ & $172(71.1)$ & $57(52.8)$ & $31(73.8)$ & & \\
\hline$\geq 2 \mathrm{~cm}$ & $18(19.4)$ & $70(28.9)$ & $51(47.2)$ & $11(26.2)$ & & \\
\hline Feature D (Diameter) & & & & & 23.094 & $<0.001$ \\
\hline$<0.5 \mathrm{~cm}$ & $78(83.9)$ & $180(74.4)$ & $59(54.6)$ & $29(69.0)$ & & \\
\hline$\geq 0.5 \mathrm{~cm}$ & $15(16.1)$ & $62(25.6)$ & $49(45.4)$ & $13(31.0)$ & & \\
\hline Feature E (Density) & & & & & 11.858 & $<0.001$ \\
\hline$<20 \mathrm{~cm}^{2}$ & $71(76.3)$ & $165(68.2)$ & $60(55.6)$ & $32(76.2)$ & & \\
\hline$\geq 20 / \mathrm{cm}^{2}$ & $22(23.7)$ & $77(31.8)$ & $48(44.4)$ & $10(23.8)$ & & \\
\hline
\end{tabular}

other; Basal vs. other). The results of the multivariate logistic regression analysis are shown in Table 3.

\section{DISCUSSION}

Breast cancer is traditionally considered as a heterogeneous disease. Most breast biopsies are performed on masses that present in mammograms as a mass or microcalcification cluster [14]. Evaluation of observed calcifications is a major assessment parameter for mammographic images. Calcifications within breast tissue are a very early sign of in situ and IDC $[15,16]$

In this study, we demonstrated associations between imaging features (related to clinicopathological parameters 
and BI-RADS 3-5 microcalcifications) and breast cancer molecular subtype. Finally, the multivariate logistic regression models show that HER-2 enriched molecular subtype is associated with calcifications with $>2 \mathrm{~cm}$ in range and calcifications with $>0.5 \mathrm{~mm}$ in diameter. In addition, multivariate analysis showed that calcification morphology (amorphour or coarse heterogenous calcifications OR: 2.847) was independently predictive of Luminal A subtype.

Amplification of HER-2 (17q21-q22) or overexpression of the HER-2 protein are considered to have prognostic and therapeutic implications. Tumors of the HER-2 enriched subtype are often aggressive and recalcitrant to treatment [13]. Although fluorescent in situ hybridization is considered to be the gold standard for detection of HER-2 gene amplification in cases with ambiguous IHC, it presents a high cost barrier because of the specialized equipment and technical expertise needed to process the sample $[17,18]$.

Mammogram calcifications are more often associated with HER-2 overexpressing tumors than with non-HER-2 overexpressing tumors. For example, Seo et al. [19] found that calcifications were more frequent in tumors with HER2 overexpression than in those without it. Patel and coworkers [20] found that patients with tumors that overexpressed HER2 were more likely to have heterogeneous and pleomorphic calcifications. However, they did not measure the range, diameter, or density of the
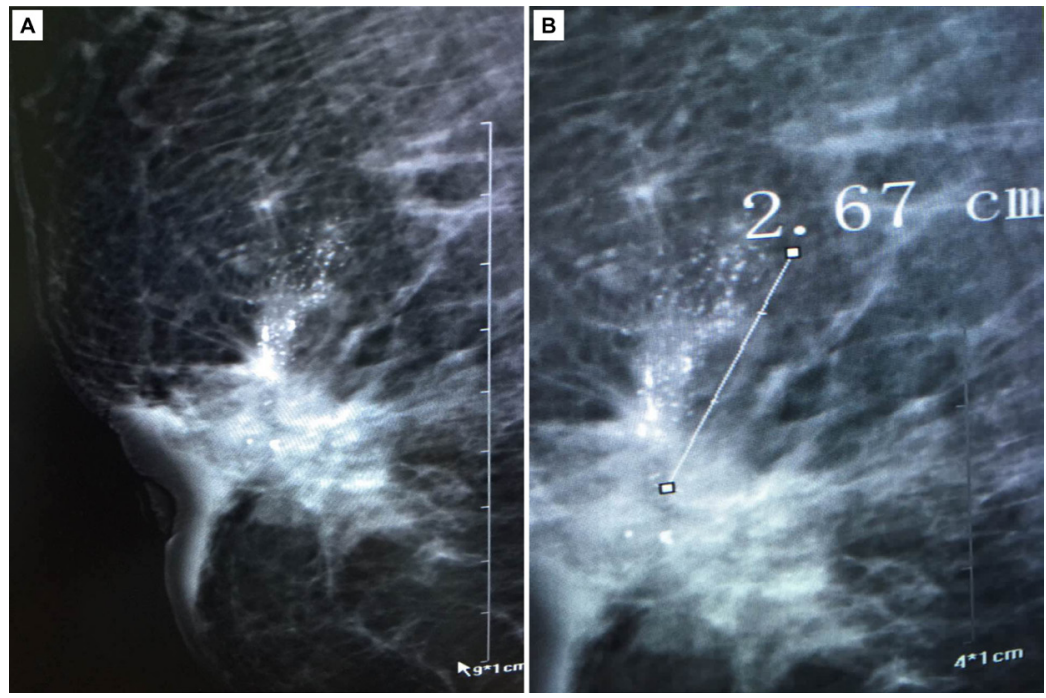

Figure 2: Infiltrating ductal carcinoma associated with microcalcification(Feature C:calcifications with $>2 \mathrm{~cm}$ in range and Feature D:calcifications with $>0.5 \mathrm{~mm}$ in diameter).

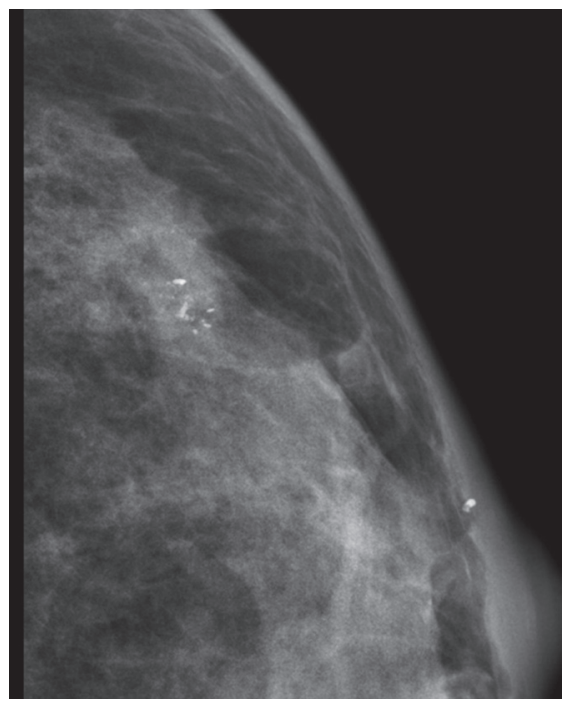

Figure 3: Infiltrating ductal carcinoma associated with microcalcification (Feature A: morphology- coarse heterogenous calcifications). 
Table 2: The correlation between mammographic imaging features and breast cancer subtype(univariate logistic regression analysis)

\begin{tabular}{|c|c|c|c|c|c|c|c|c|}
\hline & \multicolumn{2}{|l|}{ Luminal A } & \multicolumn{2}{|l|}{ Luminal B } & \multicolumn{2}{|l|}{ HER2 } & \multicolumn{2}{|l|}{ Basal } \\
\hline & Odds ratio $(95 \% \mathrm{CI})$ & Sig. & Odds ratio $(95 \% \mathrm{CI})$ & Sig. & Odds ratio $(95 \% \mathrm{CI})$ & Sig. & Odds ratio $(95 \% \mathrm{CI})$ & Sig. \\
\hline \multicolumn{9}{|l|}{ Feature A (Morphology) } \\
\hline Amorphour, Coarse heterogenous & $2.847(1.526,5.312)$ & 0.001 & Reference & & Reference & & Reference & \\
\hline Pleomorphic, Fine linear or branching, Combined & Reference & & $1.026(0.691,1.524)$ & 0.897 & $2.457(1.570,3.846)$ & $<0.001$ & $0.573(0.258,1.271)$ & 0.17 \\
\hline \multicolumn{9}{|l|}{ Feature B (Distribution) } \\
\hline Grouped or Clustered or Regional & Reference & & Reference & & Reference & & Reference & \\
\hline Linear,Segmental & $0.379(0.206,0.694)$ & 0.002 & $1.113(0.750,1.650)$ & 0.596 & $1.936(1.235,3.036)$ & 0.004 & $0.761(0.363,1.593)$ & 0.468 \\
\hline \multicolumn{9}{|l|}{ Feature C (Range) } \\
\hline$<2 \mathrm{~cm}$ & Reference & & Reference & & Reference & & Reference & \\
\hline$\geq 2 \mathrm{~cm}$ & $0.473(0.271,0.824)$ & 0.008 & $0.829(0.564,1.220)$ & 0.341 & $2.512(1.615,3.909)$ & $<0.001$ & $0.776(0.379,1.589)$ & 0.488 \\
\hline \multicolumn{9}{|l|}{ Feature D (Diameter) } \\
\hline$<0.5 \mathrm{~cm}$ & Reference & & Reference & & Reference & & Reference & \\
\hline$\geq 0.5 \mathrm{~cm}$ & $0.416(0.230,0.751)$ & 0.004 & $0.743(0.500,1.103)$ & 0.14 & $2.648(1.694,4.140)$ & $<0.001$ & $1.128(0.568,2.239)$ & 0.731 \\
\hline \multicolumn{9}{|l|}{ Feature E (Density) } \\
\hline$<20 \mathrm{~cm}^{2}$ & Reference & & Reference & & Reference & & Reference & \\
\hline$\geq 20 / \mathrm{cm}^{2}$ & $0.590(0.350,0.994)$ & 0.047 & $0.951(0.650,1.391)$ & 0.795 & $1.967(1.267,3.054)$ & 0.003 & $0.629(0.301,1.315)$ & 0.218 \\
\hline
\end{tabular}

Table 3: the correlation between mammographic imaging features and breast cancer subtype (binary logistic regression analysis)

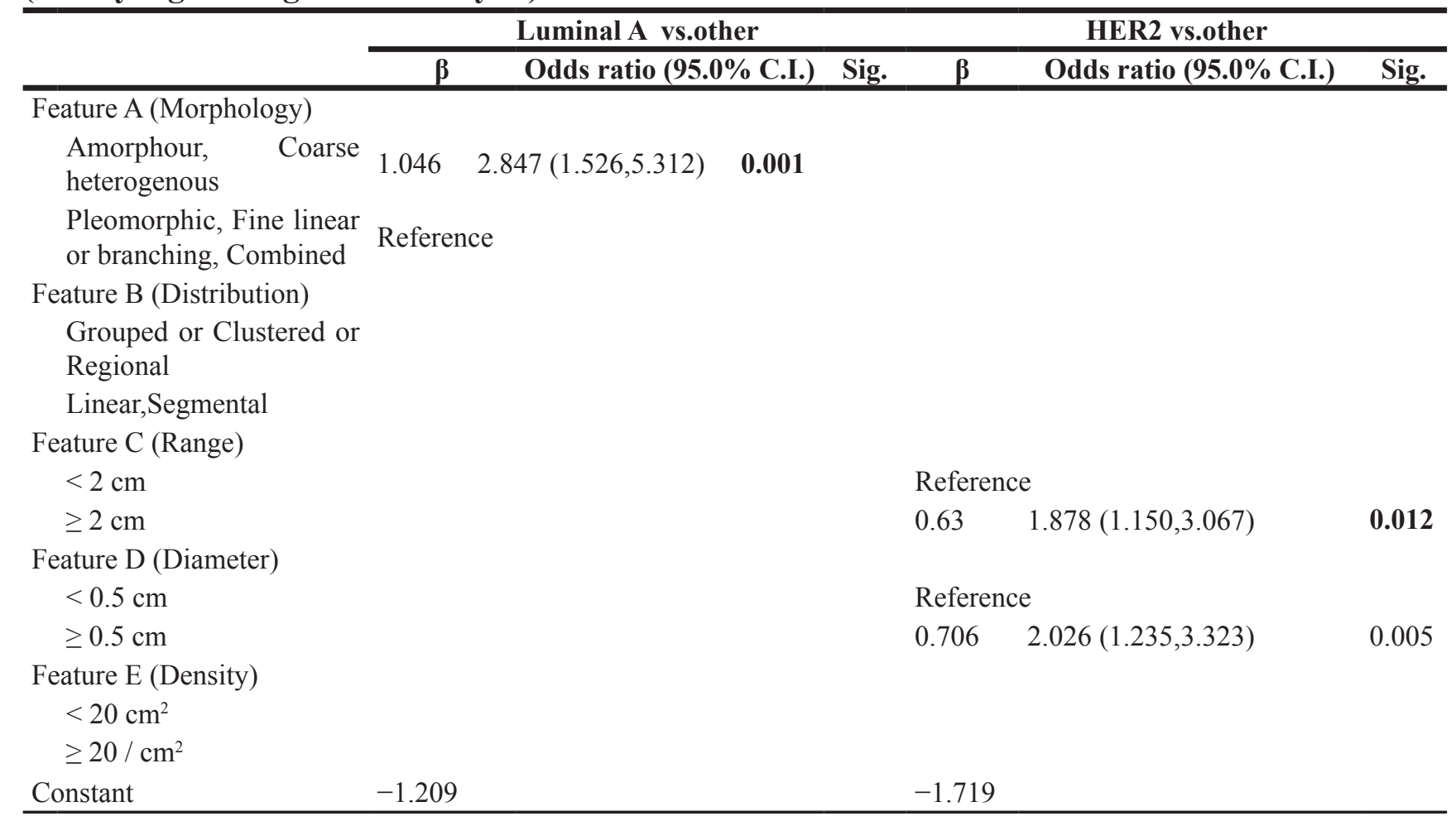

calcifications. The model showed good discrimination for predicting HER2 subtype, with a C-index of 0.704.

At the molecular level, Luminal A and B subtypes can be distinguished by the status of cell cycle-related and hormone-regulated pathways [21]. Luminal A breast cancers have better prognosis than other molecular subtypes. Luminal A cancers may also be insensitive to adjuvant chemotherapy [22]. Tamaki and coworkers [23] described several important factor divergences among luminal A tumors, such as: irregular and 
lobular versus round shape; speculated and indistinct versus microlobulated margins; amorphous versus pleomorphic calcification; and presence versus absence of cytoarchitectural distortion. In this study, we demonstrated that amorphous or coarse heterogenous calcifications were more likely to be Luminal A subtype tumor signs than were pleomorphic or fine linear or branching calcifications. The model showed good discrimination for predicting Luminal A subtype, with a C-index of 0.74 .

This study has limitations that must be acknowledged. The main limitation was the sample size with only 42 patients in the basal subtype. This small sample size may have limited the power to detect additional correlations. A further study by using a larger pool of patients of basal subtype is required.

In conclusion, our findings clearly show that mammographic calcification features can be signs of breast cancer biological features. This study presents BIRADS 3-5 microcalcifications can be conveniently used to facilitate the preoperative individualized prediction of HER2 and Luminal A molecular subtype in patients with infiltrating ductal carcinoma. This work provides useful information for pretreatment planning in breast cancer cases. Further work is needed to better define the relationships identified in our study and to explore additional relationships.

\section{MATERIALS AND METHODS}

\section{Study subjects}

The study was approved by our institutional review board, and written informed consent was obtained from all patients. Between January 2011 and April 2016, 485 consecutive patients who were referred for MG imaging of the breast and met the following inclusion criteria were respectively enrolled in our study for assessment: (1) Infiltrating ductal carcinoma (2) MG with intermediateconcern calcification or (3) MG calcifications with higher probability of malignancy. All clinical information was acquired through medical records.

\section{Mammography evaluation}

Mammographic images were analyzed using a standard four view film. All images were reviewed by two radiologists, who had 12 (Liu CL) and 7 years $(\mathrm{Xu} \mathrm{L})$, respectively, of clinical experience in the interpretation of MG imaging for the patterns of mammographically detected calcifications; discrepancies were resolved by consensus. Calcifications were classified based on the BIRADS classification system (Breast Imaging Reporting and Data System) lexicon [24 25]. We conducted a detailed image analysis to evaluate the following features of the calcifications: morphology, distribution, range, diameter and density. Calcification morphology was divided into fine branching or casting, pleomorphic or combined; the distribution was classified as grouped or clustered, linear, segmental (Feature A, morphology; Feature B, distribution). We also performed some other measurements such as range, diameter and density to more comprehensively assess the appearance of these calcifications (Feature $\mathrm{C}$, calcifications with $\leq 2 \mathrm{~cm}$ or $>2 \mathrm{~cm}$ in range; Feature $\mathrm{D}, \leq 0.5 \mathrm{~mm}$ or $>0.5 \mathrm{~mm}$ in diameter; Feature E, $\leq 20$ or $>20$ per $\mathrm{cm}^{2}$ in density) [25].

\section{Breast cancer molecular subtypes}

Four breast cancer molecular subtypes were classified by IHC based on previous reports: (1) the Luminal A subtype: ER and/or PR positive, and HER-2 negative, and Ki67 low < 14\%; (2) the Luminal B subtype: ER and/or PR positive, and HER-2 negative, and Ki67 high $\geq 14 \%$ or ER and/or PR positive and HER-2 positive; (3) the HER-2 enriched group: HER-2 positive, and ER negative, and PR negative; and the basal subgroup: ER negative, PR negative, and HER-2 negative [26-28].

\section{Heat map diagram}

Heat map was drawn to show a visual representation of gene expression trends. The amount of variation between different groups. The IHC expression of ER, PR, HER-2 and Ki-67 were plotted in a matrix by hierarchical clustering, performed by the Cluster v.3.0 program. Graphic outputs were generated by the Java TreeView v.1.6 software and presented in a color scale from green to red, where red indicated higher expression levels.

\section{Statistical analysis}

We focused on the association of mammography imaging features mentioned above (Features A-E) with specific breast cancer molecular subtypes. The risk factors were evaluated by using Chi-square test, univariate and binary logistic regression analysis.

Binary logistic regression analysis was used separately for each breast cancer subtype. In this study breast cancer molecular subtype was a binary variable (1 when a tumor belonged to the breast cancer subtype of interest, such as HER-2 enriched; and 0 if it belonged to any other molecular subtype). The logistic regression was repeated for the four breast cancer molecular subtypes: Luminal A, Luminal B, HER2 and Basal. The multivariate logistic regression models were constructed by using the binary logistic regression function in the SPSS statistical software package (version 15.0; SPSS Company, Chicago, IL). Then, the association of the imaging variables (Feature A-E) with each specific subtype was evaluated by using the likelihood ratio test function in SPSS statistical software package. Due to the small imaging variables, we included all the imaging variables (Features $\mathrm{A}-\mathrm{E}$ ). Discrimination 
was measured with the concordance index, similar to the area under the receiver operating characteristic curve: values range from 0.5 (nodiscrimination) to 1.0 (perfect discrimination) [29, 30].

\section{Abbrivations}

Features A Amorphour, Coarse heterogenous Pleomorphic, Fine linear or branching.

Features B Grouped or clustered Linear, Segmental

Features $\mathrm{C}$ Calcifications $\leq 2 \mathrm{~cm}$ in range Calcifications $>2 \mathrm{~cm}$ in range.

Features $\mathrm{D}$ Calcifications $\leq 0.5 \mathrm{~cm}$ in diameter Calcifications $>0.5 \mathrm{~cm}$ in diameter.

Features E Calcifications $\leq 20 / \mathrm{cm}^{2}$ in density Calcifications $\leq 20 / \mathrm{cm}^{2}$ in density.

\section{CONFLICTS OF INTEREST}

None.

\section{REFERENCES}

1. Siegel RL, Miller KD, Jemal A. Cancer statistics, 2016. CA Cancer J Clin. 2016; 66:7-30.

2. Celebi F, Pilanci KN, Ordu C, Agacayak F, Alco G, Ilgun S, Sarsenov D, Erdogan Z, Ozmen V. The role of ultrasonographic findings to predict molecular subtype, histologic grade, and hormone receptor status of breast cancer. Diagn Interv Radiol. 2015; 21:448-453.

3. Wiechmann L, Sampson M, Stempel M, Jacks LM, Patil SM, King T, Morrow M. Presenting features of breast cancer differ by molecular subtype. Ann Surg Oncol. 2009; 16:2705-2710.

4. Mazurowski MA, Zhang J, Grimm LJ, Yoon SC, Silber JI. Radiogenomic analysis of breast cancer: luminal B molecular subtype is associated with enhancement dynamics at MR imaging. Radiology. 2014; 273:365-372.

5. Pracella D, Bonin S, Barbazza R, Sapino A, Castellano I, Sulfaro S, Stanta G. Are breast cancer molecular classes predictive of survival in patients with long follow-up? Dis Markers. 2013; 35:595-605

6. Cho N. Molecular subtypes and imaging phenotypes of breast cancer. Ultrasonography. 2016; 35:281-288.

7. Purrington KS, Visscher DW, Wang C, Yannoukakos D, Hamann U, Nevanlinna H, Cox A, Giles GG, EckelPassow JE, Lakis S, Kotoula V, Fountzilas G, Kabisch M, et al. Genes associated with histopathologic features of triple negative breast tumors predict molecular subtypes. Breast Cancer Res Treat. 2016; 157:117-131.

8. Forero A, Li Y, Chen D, Grizzle WE, Updike KL, Merz ND, Downs-Kelly E, Burwell TC, Vaklavas C, Buchsbaum DJ, Myers RM, LoBuglio AF, Varley KE. Expression of the MHC Class II Pathway in Triple-Negative Breast Cancer
Tumor Cells Is Associated with a Good Prognosis and Infiltrating Lymphocytes. Cancer Immunol Res. 2016; 4:390-399.

9. Sutton EJ, Dashevsky BZ, Oh JH, Veeraraghavan H, Apte AP, Thakur SB, Morris EA, Deasy JO. Breast cancer molecular subtype classifier that incorporates MRI features. J Magn Reson Imaging. 2016; 44:122-129.

10. Sutton EJ, Oh JH, Dashevsky BZ, Veeraraghavan H, Apte AP, Thakur SB, Deasy JO, Morris EA. Breast cancer subtype intertumor heterogeneity: MRI-based features predict results of a genomic assay. J Magn Reson Imaging. 2015; 42:1398-1406.

11. Agner SC, Rosen MA, Englander S, Tomaszewski JE, Feldman MD, Zhang P, Mies C, Schnall MD, Madabhushi A. Computerized image analysis for identifying triplenegative breast cancers and differentiating them from other molecular subtypes of breast cancer on dynamic contrastenhanced MR images: a feasibility study. Radiology. 2014; 272:91-99.

12. Tse GM, Tan PH, Pang AL, Tang AP, Cheung HS. Calcification in breast lesions: pathologists' perspective. J Clin Pathol. 2008; 61:145-151.

13. Radenkovic S, Konjevic G, Isakovic A, Stevanovic P, Gopcevic K, Jurisic V. HER2-positive breast cancer patients: correlation between mammographic and pathological findings. Radiat Prot Dosimetry. 2014; 162:125-128.

14. Markey MK, Lo JY, Floyd CE, Jr. Differences between computer-aided diagnosis of breast masses and that of calcifications. Radiology. 2002; 223:489-493.

15. Bennett RL, Evans AJ, Kutt E, Record C, Bobrow LG, Ellis IO, Hanby A, Moss SM. Pathological and mammographic prognostic factors for screen detected cancers in a multi-centre randomised, controlled trial of mammographic screening in women from age 40 to 48 years. Breast. 2011; 20:525-528.

16. Evans AJ, Kutt E, Record C, Waller M, Bobrow L, Moss S. Radiological and pathological findings of interval cancers in a multi-centre, randomized, controlled trial of mammographic screening in women from age 40-41 years. Clin Radiol. 2007; 62:348-352.

17. Akhdar A, Bronsard M, Lemieux R, Geha S. [HER-2 oncogene amplification assessment in invasive breast cancer by dual-color in situ hybridization (dc-CISH): a comparative study with fluorescent in situ hybridization (FISH)]. Ann Pathol. 2011; 31:472-479.

18. Sauter G, Lee J, Bartlett JM, Slamon DJ, Press MF. Guidelines for human epidermal growth factor receptor 2 testing: biologic and methodologic considerations. J Clin Oncol. 2009; 27:1323-1333

19. Seo BK, Pisano ED, Kuzimak CM, Koomen M, Pavic D, Lee Y, Cole EB, Lee J. Correlation of HER-2/neu overexpression with mammography and age distribution in primary breast carcinomas. Acad Radiol. 2006; 13:1211-1218. 
20. Patel TA, Puppala M, Ogunti RO, Ensor JE, He T, Shewale JB, Ankerst DP, Kaklamani VG, Rodriguez AA, Wong ST, Chang JC. Correlating mammographic and pathologic findings in clinical decision support using natural language processing and data mining methods. Cancer. 2017; 123:114-121.

21. Prat A, Pineda E, Adamo B, Galvan P, Fernandez A, Gaba L, Diez M, Viladot M, Arance A, Munoz M. Clinical implications of the intrinsic molecular subtypes of breast cancer. Breast. 2015; 24:S26-35.

22. Pichler M, Stiegelbauer V, Vychytilova-Faltejskova P, Ivan C, Ling H, Winter E, Zhang X, Goblirsch M, WulfGoldenberg A, Ohtsuka M, Haybaeck J, Svoboda M, Gerger A, et al. Genome-wide microRNA analysis identifies miR-188-3p as novel prognostic marker and molecular factor involved in colorectal carcinogenesis. Clin Cancer Res. 2016. [Epub ahead of print]. doi: 10.1158/1078-0432. CCR-16-0497.

23. Tamaki K, Ishida T, Miyashita M, Amari M, Ohuchi N, Tamaki N, Sasano H. Correlation between mammographic findings and corresponding histopathology: potential predictors for biological characteristics of breast diseases. Cancer Sci. 2011; 102:2179-2185.

24. Nothacker M, Duda V, Hahn M, Warm M, Degenhardt F, Madjar H, Weinbrenner S, Albert US. Early detection of breast cancer: benefits and risks of supplemental breast ultrasound in asymptomatic women with mammographically dense breast tissue. A systematic review. BMC Cancer. 2009; 9:335.
25. Li JJ, Chen C, Gu Y, Di G, Wu J, Liu G, Shao Z. The role of mammographic calcification in the neoadjuvant therapy of breast cancer imaging evaluation. PLoS One. 2014; 9:e88853.

26. Cheang MC, Chia SK, Voduc D, Gao D, Leung S, Snider J, Watson M, Davies S, Bernard PS, Parker JS, Perou CM, Ellis MJ, Nielsen TO. Ki67 index, HER2 status, and prognosis of patients with luminal B breast cancer. J Natl Cancer Inst. 2009; 101:736-750.

27. Hugh J, Hanson J, Cheang MC, Nielsen TO, Perou CM, Dumontet C, Reed J, Krajewska M, Treilleux I, Rupin M, Magherini E, Mackey J, Martin M, et al. Breast cancer subtypes and response to docetaxel in node-positive breast cancer: use of an immunohistochemical definition in the BCIRG 001 trial. J Clin Oncol. 2009; 27:1168-1176.

28. Kim MS, Lee HW, Jun SY, Lee EH. Expression of alpha B crystallin and BCL2 in patients with infiltrating ductal carcinoma. Int J Clin Exp Pathol. 2015; 8:8842-8856.

29. Ho AS, Wang L, Palmer FL, Yu C, Toset A, Patel S, Kattan MW, Tuttle RM, Ganly I. Postoperative Nomogram for Predicting Cancer-Specific Mortality in Medullary Thyroid Cancer. Ann Surg Oncol. 2015; 22:2700-2706.

30. Chok AY, Goh BK, Koh YX, Lye WK, Allen JC, Jr., Quek R, Teo MC, Chow PK, Ong HS, Chung AY, Wong WK. Validation of the MSKCC Gastrointestinal Stromal Tumor Nomogram and Comparison with Other Prognostication Systems: Single-Institution Experience with 289 Patients. Ann Surg Oncol. 2015; 22:3597-3605. 